\title{
Hemophagocytic Lymphohistiocytosis Secondary to Dermatopathic Lymphadenitis
}

\author{
Yingying WANG ${ }^{*}$, Shangqin $\mathrm{LIU}^{1}$, Yong $\mathrm{HE}^{2}$, Liang $\mathrm{SHAO}^{1}$ and Guifang YANG
}

${ }^{1}$ Department of Hematology, Zhongnan Hospital of Wuhan University, Wuhan, Hubei, China

${ }^{2}$ Department of Nuclear medicine, Zhongnan Hospital of Wuhan University, Wuhan, Hubei, China

${ }^{3}$ Department of Pathology, Zhongnan Hospital of Wuhan University, Wuhan, Hubei, China

${ }^{*}$ Corresponding author: Yingying WANG, M.D, Department of Hematology in Zhongnan Hospital of Wuhan University, 169 Donghu Road, Wuchang, Wuhan, Hubei 430071, P.R, China, Tel: 008615623696683, E-mail: yingyingvictoria@hotmail.com

Citation: Yingying WANG, Shangqin LIU, Yong HE, Liang SHAO, Guifang YANG (2019) Hemophagocytic Lymphohistiocytosis Secondary to Dermatopathic Lymphadenitis. J Case Rep Stud 7(1): 103. doi: $10.15744 / 2348-9820.7 .103$

Received Date: December 26, 2018 Accepted Date: February 25, 2019 Published Date: February 27, 2019

\begin{abstract}
Dermatopathic lymphadenitis (DL) is considered a benign disease, the symptoms of which can be relieved with only anti-histamine. It can be confused with lymphoma. Here, we report a case of DL, which resulted in hemophagocytic lymphohistiocytosis (HLH). After adequate treatment, remission was obtained at first; however, recurrence with liver failure occurred six months later, which quickly resulted in death. This particular case has not been reported elsewhere. It reminds us that even benign dermatological problems can cause life-threatening syndromes, such as, in this case, HLH. The risk needs to be taken seriously in patients presenting with fever, skin problems, and lymphadenopathy.
\end{abstract}

Keywords: Hemophagocytic Lymphohistiocytosis; Dermatopathic Lymphadenitis; Skin Problems; Liver Dysfunction

\section{Introduction}

Dermatopathic lymphadenitis (DL) is a rare benign disease. It was first described by Pautrier and Woringer as lipomelanotic reticulosis, which represents a rare form of benign lymphatic hyperplasia associated with most exfoliative or eczematoid inflammatory erythrodermas, including pemphigus, psoriasis, eczema, neurodermatitis, and atrophia senilis [1]. It triggers a distinctive reaction pattern in lymph nodes, characterized by paracortical hyperplasia composed of interdigitating dendritic cells, Langerhans cells, macrophages containing melanin pigment, and small T cells. It was named DL in 1942 by Hurwitt, and it is believed to represent an exaggerated response to increased antigenic stimulation in the skin [2,3]. Typical features are pale cortical areas with deposits of melanin in the periphery of the lymph nodes and predominantly para-cortical proliferation of benign histiocytes, which are often found to be CD68-positive and S100-positive in immunochemistry experiments [4]. This disease could be easily confused with mycosis fungoides (Sézary syndrome). Hence, during diagnosis, care should be taken to discern between DL and malignant lymphoma [5].

Hemophagocytic lymphohistiocytosis (HLH) is a life-threatening disease caused by a dysregulated immune reaction. Primary HLH is caused by defects in several genes, including PRF1, UNC13D, STX11, STXBP2, RAB27A, LYST, and AP3B1. Secondary HLH or acquired HLH involves an uncontrolled immune reaction caused by infections, malignancies, or autoimmune diseases [6]. Over activation of immune cells like macrophages, neutrophils, and monocytes results in cytokine overproduction, this causes a series of inflammatory reactions [7]. Its typical features include fever, hepatosplenomegaly, cytopenia, and hematophacocytic phenomena in hematopoietic organs [8]. According to the Consensus Statements by the HLH Steering Committee of the Histiocyte Society in 2018, Etoposide-based protocols are still a valuable option for the treatment of HLH [9].

Here, we present one case of HLH associated with DL. The patient firstly presented with skin lesions; later she also had swollen lymph nodes and fever. Although DL is considered a benign disease, this patient also developed HLH. Remission was obtained after adequate treatment firstly; however, recurrence with liver failure occurred six months later, which quickly resulted in death.

\section{Case report}

A 40-year-old woman with a history of urticaria and itching skin for about 2 months was admitted to our hospital because of intermittent fever for about 3 weeks. She reported that her symptoms of skin rash and itching started two months ago. She 
systematically took steroids and locally applied ointment for about one month after visiting a dermatologist, and all symptoms were relieved. Three weeks before admittance, she had a fever after catching a cold. The highest temperature she reported was $39.5^{\circ} \mathrm{C}$, with chill before fever, without other infectious symptoms like cough, diarrhea, or headache. She was given an antibiotic treatment with cephalosporins in a local hospital, but her fever did not resolve, and she progressively developed generalized lymphadenopathy. Hence, she was transferred to the Zhongnan Hospital of Wuhan University. Her temperature was $36.6{ }^{\circ} \mathrm{C}$ at the moment of admission. Physical examination revealed scattered non-blanchable erythematous papules coalescing into plaques, most prominently in the upper chest and bilateral upper extremities. Generalized lymphadenopathy was observed, with the largest lymph nodes measuring up to $2 \mathrm{~cm}$ in diameter. The lymph nodes were firm, non-tender, and mobile. She showed no hepatosplenomegaly.

Laboratory tests revealed the following: white blood cell count of $7.29 \times 10^{9}$ cells/L (normal range: $3.5-9.5 \times 10^{9}$ cells/L), red blood cell count of $3.75 \times 10^{12}$ cells/L (normal range: $3.8-5.1 \times 10^{9}$ cells $/ \mathrm{L}$ ), hemoglobin concentration of $110.3 \mathrm{~g} / \mathrm{L}$ (normal range: $110-$ $150 \mathrm{~g} / \mathrm{L}$ ), platelet count of $169 \times 10^{9}$ cells/L (normal range: $125-350 \times 10^{9}$ cells/L), C-reactive protein concentration of $13.23 \mathrm{mg} / \mathrm{L}$ (normal range: $0-10 \mathrm{mg} / \mathrm{L}$ ), procalcitonin concentration of $0.09 \mathrm{ng} / \mathrm{mL}$ (normal range: $0-0.05 \mathrm{ng} / \mathrm{mL}$ ), IL-6 concentration of 19.57 $\mathrm{ng} / \mathrm{mL}$ (normal range: $0-7 \mathrm{ng} / \mathrm{ml}$ ), erythrocyte sedimentation rate of $38 \mathrm{~mm} / \mathrm{h}$ (normal range: $0-20 \mathrm{~mm} / \mathrm{h}$ ), ferritin concentration of $24771.76 \mathrm{ng} / \mathrm{mL}$ (normal range: $4.63-204.00 \mathrm{ng} / \mathrm{mL}$ ), immunoglobulin A concentration of $4.41 \mathrm{~g} / \mathrm{L}$ (normal range: $0.7-4.0 \mathrm{~g} / \mathrm{L}$ ), immunoglobulin G concentration of $15.7 \mathrm{~g} / \mathrm{L}$ (normal range: 7-16 g/L), immunoglobulin M concentration of $2.04 \mathrm{~g} / \mathrm{L}$ (normal range: $0.40-2.30 \mathrm{~g} / \mathrm{L}$ ), immunoglobulin E concentration of $215 \mathrm{IU} / \mathrm{mL}$ (normal range: $0-100 \mathrm{IU} / \mathrm{mL}$ ), C3 concentration of 0.99 $\mathrm{g} / \mathrm{L}$ (normal range: $0.90-1.80 \mathrm{~g} / \mathrm{L}$ ), C4 concentration of $0.204 \mathrm{~g} / \mathrm{L}$ (normal range: $0.10-0.40 \mathrm{~g} / \mathrm{L}$ ), and lactate dehydrogenase (LDH) concentration of $895 \mathrm{U} / \mathrm{L}$ (normal range: 125-243.0 U/L). Serology tests for various infections, including cytomegalovirus (CMV) and Epstein Barr virus (EBV), were negative (tests included (i) IgA and IgM anti-EBV viral capsid antigen and (ii) IgA and IgM anti-CMV). NK cell activity was evaluated by flow cytometry. The proportion of CD3+ CD56+ NK cells of the patient's lymphocytes was $2.32 \%$ (normal range: $5 \%-26 \%$ ), granzyme B activity was $94.80 \%$ (normal range: $>78 \%$ ), perforin activity was $61.27 \%$ (normal range: $>84 \%$ ), and the soluble CD25 concentration was $15383 \mathrm{pg} / \mathrm{mL}$ (normal range: $400-2700 \mathrm{pg} / \mathrm{mL}$ ). Bone marrow aspiration demonstrated infectious phenomena with $1 \%$ of abnormal and suspicious lymphoma cells. Serology tests for HIV, HBV, and HCV were negative. Tests for tuberculosis such as the PPD tuberculin skin test and tuberculin T-spot were negative. Blood culture, urine culture, and marrow culture results were negative. Due to the clinical suspicion of lymphoma, ${ }^{18} \mathrm{~F}-$ fluorodeoxyglucose $\left({ }^{18} \mathrm{~F}-\mathrm{FDG}\right)$ positron emission tomography/computed tomography (PET/CT, Siemens Biograph mCT) were performed after the intravenous injection of $236-\mathrm{MBq}{ }^{18} \mathrm{~F}-\mathrm{FDG}$. Imaging results revealed intensive focal uptake in multiple enlarged lymph nodes and diffuse uptake in the spleen and bone marrow (Figure 1).
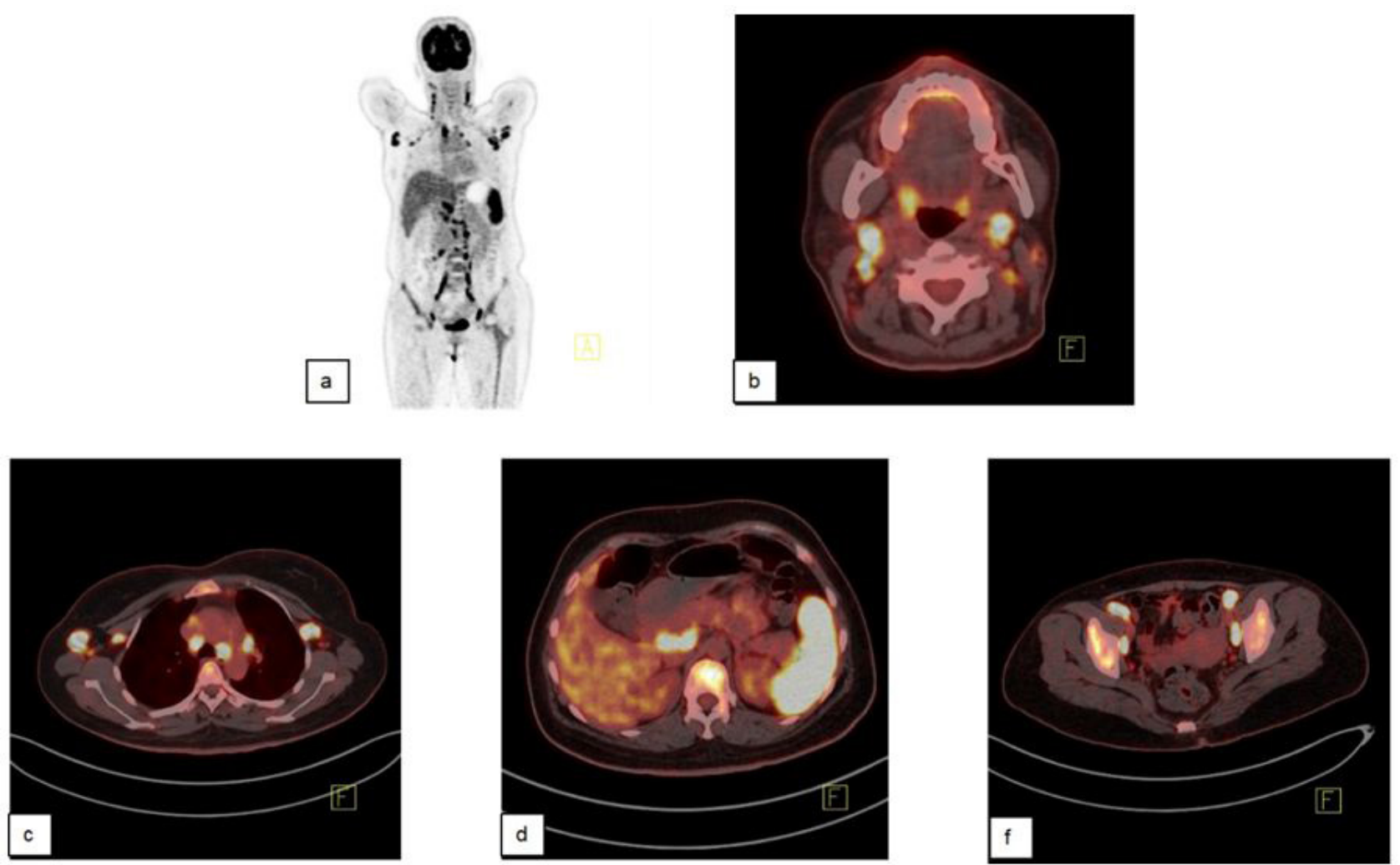

Figure 1: ${ }^{18} \mathrm{~F}-\mathrm{FDG}$ PET/CT images of the patient with dermatopathic lymphadenitis. (a) Maximum intensity projection positron emission tomography image shows extensive ${ }^{18} \mathrm{~F}-\mathrm{FDG}$-avid lymphadenopathy in bilateral cervical, supraclavicular, axillary, and mediastinal, abdominal, pelvic, and inguinal regions and diffuse uptake in spleen and bone marrow. (b-f) Transaxial PET/CT fusion images show enlarged cervical, axillary, abdominal, iliac lymph nodes. The largest lymph node measures $25 \mathrm{~mm} \times 10 \mathrm{~mm}$ in axillary area with maximum standardized uptake value of 14.4 
Journal of Case Reports and Studies

Right cervical lymph node biopsy was performed, and pathology experiments revealed lymphoid hyperplasia along with normal lymphoid tissue. Immunohistochemical analysis showed the following. The lymphoid follicle area was CD20(+), CD21 (FDC reticule +), and CD23(+); the paracortical zone was CD68(+), CD163(+), S100(+), CD2(+), CD3(+), CD5(+), CD7(+), CD4(+), CD8(+), PD-1(-), GranzyB(-), ALK(-), CD56(-), CD30(-), CD10(-), Bcl-6(-), Ki-67(+,30\%), MPO(-), CD123(-), Langerhans cell with Langerin $(+)$, and CD1a(+). EBV-encoded small nuclear RNA-positive mononuclear cells were not detected in tissue samples by FISH (Figure 2).

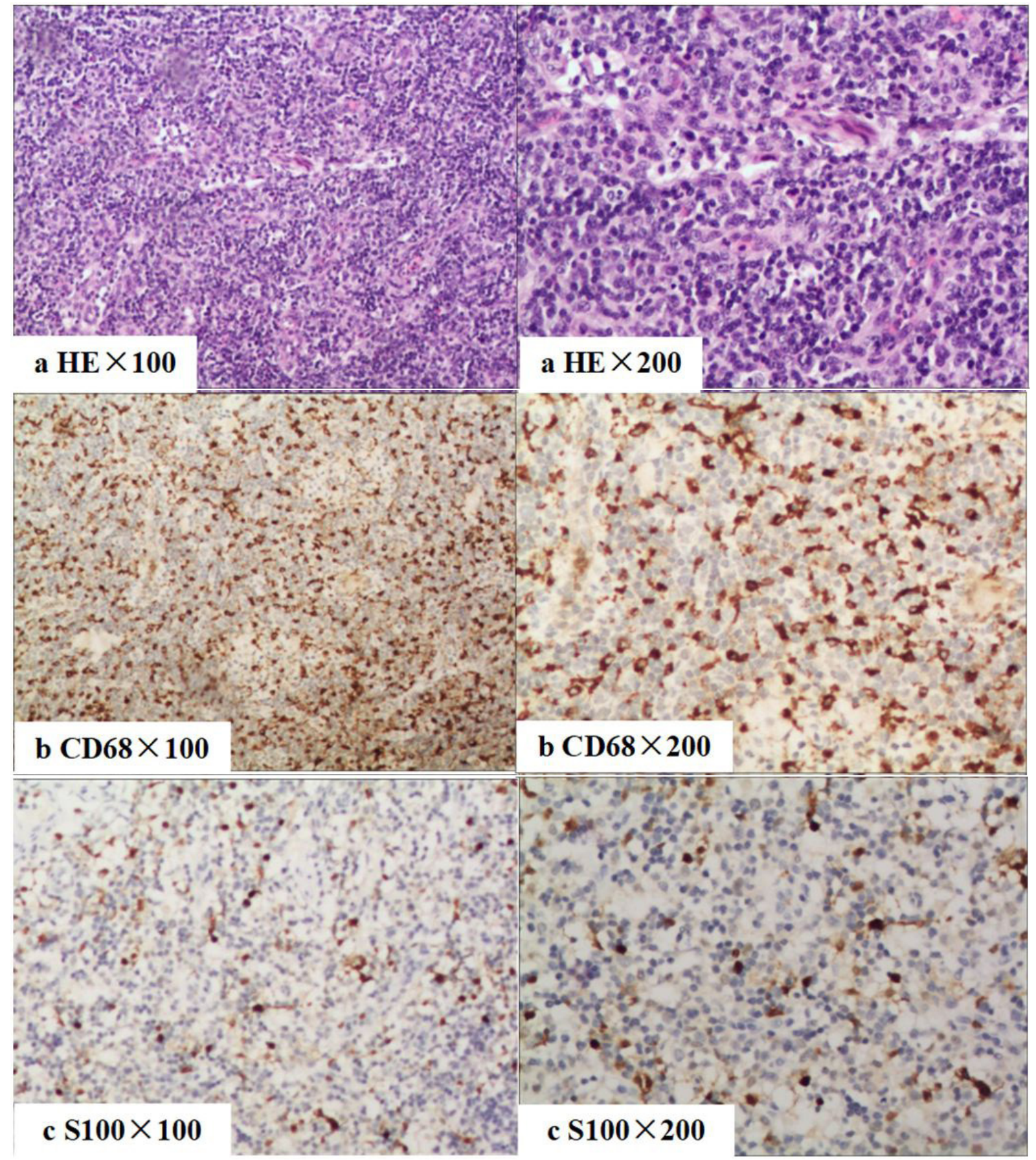

Figure 2: Right cervical lymph node revealed pale-stained areas of histiocytic aggregates without cellular atypia in the cortex. The histiocytes contained abundant pinkish to amphophilic cytoplasm with centrally located nuclei (Figure 2a left: $\times 100$; right: $\times 200$ ) and immunohistochemical analysis revealed CD68 positivity (Figure $2 \mathrm{~b}$ left: $\times 100$; right: $\times 200$ ) and S100 positivity (Fig. $2 c$ left: $\times 100$; right: $\times 200)$. and these findings were consistent with DL

With all the above evidence, the following diagnosis was made: HLH secondary to DL. We chose to treat the patient following the HLH 2004 protocol with etoposide (VP-16) and dexamethasone. A reduced VP-16 dose of 100 mg per day was given for 3 days. Dexamethasone was administered firstly at a dose of $15 \mathrm{mg}$ daily for two weeks. Due to poor patient compliance, etoposide treatment was stopped after the first three doses, and the corticoid was prescribed orally. The symptoms were relieved without fever, and she recovered with normalization of ferritin levels, LDH levels, and liver function. 
NK cell activity was again evaluated one month later. The proportion of CD3+ CD56+ NK cells in lymphocytes was $10.62 \%$ (normal range: 5\%-26\%), granzyme B activity was 96.49\% (normal range: $>78 \%$ ), perforin activity was $97.43 \%$ (normal range: $>84 \%$ ), and a concentration of soluble CD25 of $3184 \mathrm{pg} / \mathrm{mL}$ (normal range: $400-2700 \mathrm{pg} / \mathrm{mL}$ ) was measured.

She stopped steroid therapy by herself after having taken steroids for about four months. In the sixth month, she had a skin rash and itching skin again. She visited the dermatologist and restarted with steroid therapy, but the symptoms did not improve. Furthermore, she got fever and liver dysfunction with elevated alanine transaminase (ALT) and aspartate transaminase (AST) levels. Hence, she was again hospitalized. The biological tests indicated recurrence of HLH. While more evidence was sought, VP16 plus dexamethasone treatment was started immediately. However, her symptoms were not relieved, and her liver function worsened. The second line of treatment was given with intravenous immunoglobulin, this did not work either. She suffered liver failure quickly, with a high ALT concentration of $569 \mathrm{U} / \mathrm{L}$, AST concentration of $251 \mathrm{U} / \mathrm{L}$, total bilirubin concentration of $361.1 \mu \mathrm{mol} / \mathrm{L}$ (normal range: $5-21 \mu \mathrm{mol} / \mathrm{L}$ ), direct bilirubin concentration of $214.1 \mu \mathrm{mol} / \mathrm{L}$ (normal range: $0-7 \mu \mathrm{mol} / \mathrm{L}$ ), indirect bilirubin concentration of $214.1 \mu \mathrm{mol} / \mathrm{L}$ (normal range: $1.5-18 \mu \mathrm{mol} / \mathrm{L}$ ), albumin (ALB) concentration of 27.2 g/L, globulin (GLB) concentration of $35.8 \mathrm{~g} / \mathrm{L}$, disturbance of coagulation system with prolonged PT of $19.3 \mathrm{~s}$ (normal range: 9.4-12.5s), and an APTT of 42.7 s (normal range: 25.1-36.5 s). Serology tests of hepatitis A, B, C, D, and E were negative. The symptoms aggravated, with further liver dysfunction and disturbance of the coagulation system, and she died within one week.

\section{Discussion}

As described previously, this patient presented skin symptoms at first, followed by the occurrence of fever and lymphadenopathy. Biopsy of the cervical lymph nodes showed typical hyperplasia features, and immunochemistry staining showed that histiocytes were CD68-positive, S100-positive, and CD1a-positive. Serology tests were negative for CMV, EBV, HIV, and hepatitis, and no other infections were reported. Furthermore, this patient had fever with liver dysfunction, hyperferritinemia, elevated serum levels of soluble CD25, and lower NK cell activity. The diagnosis was clearly HLH secondary to DL. It seems not logical that DL can cause hemophagocytic lymphohistocytosis, because DL is usually considered a benign disease which could be relieved spontaneously with only anti-histamine treatment. However, DL and HLH were confirmed in this patient, and we did not find other causes for HLH, so we suspect HLH resulted from DL. Therefore, in clinical practice, caution must be practiced when a patient presents with skin disease, constant fever, and lymphadenopathy. A life-threatening syndrome such as HLH could be triggered, so this must be prevented. In 2010, a case of EBV-associated HLH was reported with concomitant DL and more cases of a cutaneous manifestation in HLH patients have been reported [10]. It is plausible that there is a link between HLH and DL, as both diseases involve the immune system; HLH is associated with the systemic immune reaction and DL with local immune reactions, especially skin reactions. If local immune cells are over activated, this could trigger a systemic immune reaction. Further research is required to investigate the underlying pathogenesis of these two diseases.

The ${ }^{18} \mathrm{~F}-\mathrm{FDG}$ PET/CT imaging results from this study indicate the patient had multiple hypermetabolic lymph nodes and increased FDG uptake in bone marrow and the spleen. Both DL and lymphoma appear to increase the uptake in ${ }^{18} \mathrm{~F}-\mathrm{FDG}$ PET/ CT experiments. Diffusely increased splenic and bone marrow uptake is more often seen in lymphoma infiltration, inflammation/ infection, autoimmune disorders, and HLH. The reason why we observed an increased uptake in the DL/HLH patient may be that ${ }^{18} \mathrm{~F}-\mathrm{FDG}$ is absorbed by activated immune cells, including T lymphocytes and macrophages. ${ }^{18} \mathrm{~F}-\mathrm{FDG}$ PET/CT is not specific for malignant lymphomas. However, PET/CT can guide us to carry out lymph node-specific and bone marrow-specific biopsy. There are two reports available on the application of PET/CT scans in DL patients. Both showed DL patients had hypermetabolic lymph nodes, mimicking lymphoma [11,12]. PET/CT, a sensitive and non-invasive whole-body imaging technique, can be used as a valuable aid in diagnostics.

The case presented in this study has several implications. Firstly, all HLH cases, even those secondary to a benign disease, need to be treated as seriously as other cases of secondary HLH. The patient in our study showed remission after the first cycle of therapy with etoposide and dexamethasone, so she was discharged and she continued the steroid therapy without etoposide. The recurrence could possibly be ascribed to her poor compliance during ectoposide and corticoid treatment. Secondly, this patient presented with liver dysfunction initially and at the moment of recurrence. Liver biopsy might have been useful, particularly at the moment of recurrence, to find specific abnormalities or to give her a specific treatment. Finally, no genetic tests were carried out for this patient. Although the patient did not have a family history of HLH and she presented skin problems prior to the occurrence of HLH, potential genetic defects were not screened for. If a genetic defect had been found, standard treatment could have protected her from recurrence, saving her life. In summary, HLH can have many other causes; even a benign disease, such as $\mathrm{DL}$, which could cause a severe immune reaction and trigger the development of HLH, should be taken as seriously as other cases of secondary HLH.

\section{Conclusion}

This is a rare case of HLH secondary to DL. The diagnosis, based on lymph node pathology and laboratory tests, left no room for doubt. Although the patient initially showed remission, which lasted for about 6 months, the recurrence resulted in her death. Her treatment could have been improved by (i) evaluating EBV-DNA frequently, (ii) doing genetic tests to rule out familial HLH, 
(iii) liver biopsy, and (iv) respecting the standard protocol. We share this case aiming to warn others who treat patients presenting with hemophagocytic syndrome. HLH could be fatal, regardless of the underlying cause, and it is always worthy of serious medical attention, rigorous treatment conforming to the standard HLH treatment protocol, and close medical follow-up.

\section{Acknowledgments}

Yingying WANG collected the information on this case and prepared this manuscript; Shangqin LIU and Liang SHAO revised the draft; Yong $\mathrm{H}$ was responsible for the part about the interpretation of PET-CT scan results and provided the PET-CT scan images; Guifang YANG was responsible for the part about pathology interpretation and provided the lymph node pathology images. This work was supported by a grant from the fundamental research funds from the central universities.

\section{References}

1. Steffen C (2004) Pautrier-Woringer disease (lipomelanotic reticulosis/dermatopathic lymphadenitis). Am J Dermatopathol 26: $499-503$.

2. Cribier B (2005) History: Frederic Woringer (1903-1964) and Woringer-Kolopp disease. Am J Dermatopathol 27: 534-45.

3. Gould E, Porto R, Albores-Saavedra J, Ibe MJ (1988) Dermatopathic lymphadenitis. The spectrum and significance of its morphologic features. Arch Pathol Lab Med 112: 1145-50.

4. Shaposhnikov OK, Genter EI, Rodionov AN (1981) [Histological and immunological characteristics of dermatopathic lymphadenitis]. Vestn Dermatol Venerol 1: $4-7$.

5. Winter LK, Spiegel JH, King T (2007) Dermatopathic lymphadenitis of the head and neck. J Cutan Pathol 34: 195-7.

6. Daver N, McClain K, Allen CE, Parikh SA, Otrock Z, et al. (2017) A consensus review on malignancy-associated hemophagocytic lymphohistiocytosis in adults. Cancer 123: 3229-40.

7. Brisse E, Wouters CH, Matthys P (2015) Hemophagocytic lymphohistiocytosis (HLH): A heterogeneous spectrum of cytokine-driven immune disorders. Cytokine Growth Factor Rev 26: 263-80.

8. Schram AM, Berliner N (2015) How I treat hemophagocytic lymphohistiocytosis in the adult patient. Blood 125: 2908-14.

9. Ehl S, Astigarraga I, von Bahr Greenwood T, Hines M, Horne A, et al. (2018) Recommendations for the Use of Etoposide-Based Therapy and Bone Marrow Transplantation for the Treatment of HLH: Consensus Statements by the HLH Steering Committee of the Histiocyte Society. J Allergy Clin Immunol Pract 6: 1508-17.

10. Lee WJ, Lee DW, Kim CH, Won CH, Chang SE, et al. (2010) Dermatopathic lymphadenitis with generalized erythroderma in a patient with epstein-barr virus-associated hemophagocytic lymphohistiocytosis. Am J Dermatopathol 32: 357-61.

11. Makis W, Hickeson M, Blumenkrantz M (2010) Interesting image. Dermatopathic lymphadenitis: a pitfall for lymphoma evaluation by F-18 FDG PET/CT. Clin Nucl Med 35: 872-4.

12. Hu N, Tan YL, Cheng Z, Wang YH (2015) Dermatopathic Lymphadenitis. Chin Med J 128: 3121-2. 